\title{
ANÁLISE DAS FRAÇÕES DE VAZIOS DE UM ESCOAMENTO BIFÁSICO (AR-AGUA) VERTICAL COM SONDAS CAPACITIVAS
}

\author{
G. F. SILVA ${ }^{1}$, e A. L. O. FERREIRA ${ }^{1}$ \\ ${ }^{1}$ Instituto Federal da Bahia, Engenharia Ambiental e Sanitária
}

\begin{abstract}
RESUMO - Muitas aplicações industriais envolvem fenômenos com duas ou mais fases. Entre estes, o escoamento bifásico de líquido e gás representa um papel importante na indústria petrolífera, assim como em reatores nucleares e na indústria química. Este trabalho apresenta um estudo experimental sobre a técnica de medição capacitiva das frações volumétricas "in situ" das fases em escoamento multifásico. Busca-se desenvolver uma sonda não intrusiva de fácil montagem e a baixo custo, mas que seja capaz de fornecer dados instantâneos sobre a distribuição de ar, e água numa seção transversal qualquer do tubo, atentando-se para determinação as frações de vazio no escoamento bifásico vertical ar-água através de sondas capacitivas. Portanto, foram utilizadas duas técnicas para analisar o sinal da sonda, os modelos usados foram não lineares do tipo NARX polinomiais e redes neurais, as duas técnicas indicaram que o uso desse tipo de técnica capacitiva, permite a determinação das frações de vazio, desde que bem calibradas. De acordo com os dados obtidos, tanto o método NARMAX polinomial e a rede neural apresentaram bom resultados.
\end{abstract}

\section{INTRODUÇÃO}

Escoamentos bifásicos em tubulações podem assumir diferentes configurações geométricas que foram divididas em alguns padrões básicos de acordo com o arranjo de cada fase (Wallis, 1969). Ao longo do percurso, as configurações do escoamento podem ser alteradas, passando de um padrão para outro, ou seja, poderá haver uma sucessão de padrões. O tipo de padrão em cada trecho do duto depende de condições operacionais (vazão, pressão, temperatura, velocidade do líquido e velocidade do gás), condições geométricas da tubulação (dimensão, inclinação e forma) e de propriedades das fases (densidade e viscosidade). As classificações dos padrões de escoamento são concordantes entre diferentes pesquisadores (Hewitt, (1982); Matsui, (1986); Taitel et al., (1980); Hubbard e Dukler (1966), França (1987),

Os padrões para o escoamento bifásico vertical ascendente são classificados em três grupos denominados por Bolhas, Intermitente e Anular. Por sua vez cada padrão foi dividido em sub-padrões. O padrão Bolhas engloba os sub-padrões de bolhas em regime esférico e mono-dispersas e o capa esférica (spherical cap). O padrão Intermitente engloba os arranjos onde há a alternância de bolhas de gás que ocupam toda a seção transversal do tubo e pistões de líquido. Ele foi dividido nos subpadrões: golfadas de líquido (golfadas) e golfadas instáveis (agitado). Por último vem o padrão Anular que foi dividido nos sub-padrões semi-anular e anular. 


\section{9 a 22 de outubro de 2014 \\ Florianópolis/SC}

De forma geral, podem ser categorizados como intrusivos (ou de baixa impedância), quando o sistema tem qualquer influência sobre o escoamento, mesmo quando não ocorre contato direto entre a sonda ou sensor e o fluido; e não-intrusivos (ou de alta impedância), que não influem de forma alguma na dinâmica do escoamento. Os capacitivos possuem algumas vantagens: não necessitam de proteção e cuidados especiais durante a operação do sistema, podem operar com fluidos opacos ou sujos, uma alta sensibilidade pode ser alcançada durante a etapa de projeto do circuito transdutor, são não-intrusivos, estáveis e de custo reduzido.

Um sistema de medida de fração de vazio capacitivo é composto de duas partes: um sistema de eletrodos (eletrodos fonte e sensor, com sistema de guarda e blindagem) e um circuito transdutor de capacitância. A fração de vazio é medida por um par de placas ou eletrodos instalados na parede externa do tubo e que detectam a mudança da permissividade dielétrica média do meio entre eles. Como o gás e o líquido possuem propriedades dielétricas distintas, o sinal de saída do transdutor é proporcional à quantidade das fases na seção de medida.

Os sinais adquiridos não são diretamente utilizados pelas metodologias desenvolvidas. Esses sinais variam bastante devido ao arranjo espacial das fases de gás e líquido, de modo que se torna difícil identificar o padrão diretamente a partir deste sinal. Desta forma, todos os trabalhos fazem algum tipo de transformação para os sinais nas metodologias propostas.

Existem diferentes técnicas para o desenvolvimento de sensores de software. A técnica mais utilizada recentemente são as redes neurais artificiais, como pode ser observado em muitas publicações. Alguns trabalhos mostram que os sensores baseados em redes neurais apresentam resultados mais precisos e uma maior capacidade de reconhecer padrões em sistemas complexos, não lineares e com atrasos, em comparação com outras tecnologias como "fuzzy" e "neuro-fuzzy", Linko, Zhu e Linko, (1999), Araúzo-bravo et.al. (2004), Assis e Maciel (200).

É possível observar na literatura que diversas técnicas são empregadas para a identificação de modelos utilizados pelos Sensores Virtuais (Soft Sensors). A mais simples de todas estas técnicas é a identificação linear paramétrica que utiliza modelos compostos por somatórias de funções simples ponderadas por parâmetros, que podem ser encontrados através de técnicas de regressão tal como somatória dos mínimos quadrados. Modelos utilizados para essa identificação são do tipo entradasaída tais como ARMA, ARX, NARX, ARMAX e NARMAX. Segundo Aguirre (2007), mais do que melhorar a exatidão dos modelos, o uso de representações não-lineares e necessária por possibilitar a representação de certos comportamentos dinâmicos que não são possíveis de serem descritos com o modelos lineares.

Os modelos NARMAX, sigla de não-linear auto regressivo com media móvel e entradas exógenas (non-linear autoregressive moving average model with exogenous inputs), podem ser representado da seguinte maneira: 
$\mathrm{y}(k)=F\left[y(k-1), \ldots, y\left(k-n_{y}, u\left(k-1, \ldots, u\left(k-n_{u}, e(k), e(k-1, \ldots e(k-\right.\right.\right.\right.$ $\left.\left.\left.\left.\left.n_{e}\right)\right)\right)\right)\right]$

Um modelo NARMAX polinomial é uma expansão da função $F$ da Equação 1 em uma função polinomial com grau de não-linearidade . em $\mathrm{t}=1, \ldots, \mathrm{N}$. F é uma função polinomial qualquer com grau de não linearidade $1 . y(t), u(t)$ e e(t) são, respectivamente, saída, entrada e ruído do sistema, cujos atrasos máximos são representados por ny, nu, e ne, respectivamente. $\mathrm{k}$ representa o tempo morto do sistema e $\mathrm{N}$ é o número de pontos dos dados de identificação.

Considera-se que o sistema não tem atraso puro de tempo e que nenhum dos parâmetros a ser estimado depende de e(k). Assim, um modelo NARMAX polinomial é dado por uma parte determinística NARX, mais uma parte media móvel contendo o erro. No modelo NARMAX (assim como no NARX), a função $F$ pode ser modelada por um modelo polinomial a aproximação polinomial de grau 1 e descrita pela Equação 2:

$$
y(k)=\sum_{m=0}^{l} \sum_{p=0}^{m} \sum_{n_{1}, n_{m}} c_{p, m-p}\left(n_{l}, K, n_{m}\right) \prod_{i=1}^{p} y\left(k-n_{i}\right) \prod_{i=p+1}^{m} u\left(k-n_{i}\right) \prod_{q=1}^{n_{e}} e(k-q)
$$

Ou seja, $y(k)$ é uma soma de termos com grau de não linearidade $\mathrm{m}$, com $1 \leq \mathrm{m} \leq$. Sendo $\mathrm{p} \mathrm{o}$ grau de y e q o grau de $u$ num dado termo, então $\mathrm{p}+\mathrm{q}=\mathrm{m} \leq 1$.

\section{METODOLOGIA}

A etapa experimental foi constituída das seguintes fases:

Primeira etapa:

Montagem do aparato experimental: Um conjunto experimental foi projetado e construído especificamente para o estudo de medições com sondas capacitivas de frações de vazio em escoamentos bifásicos água-ar verticais para cima. O sistema é composto dos seguintes acessórios:

I.Dois tanques de $10 \mathrm{~L}$ para armazenamento;

II. Uma bomba;

III. Um pequeno compressor; 
IV. Uma tubulação de $2 \mathrm{~m}$, com diâmetro interno de 29,5mm e externo de 39,85mm, válvulas, tês, reduções, curvas;

V. Tubos de acrílico com $300 \mathrm{~mm}$ de comprimento, com diâmetro interno de 29,5mm e externo de 39,85mm;

VI. Sensor capacitivos;

VII. Montagem do sistema de aquisição de dados.

Segunda etapa: 1.

Experimentação: O sensor capacitivo foi instalado no tubo de acrílico como mostrado na Figura

O processo de medição das capacitâncias foi feito com a medição monofásica, primeiramente para a água; logo após os tubos eram limpos e a medição para o ar era efetuada. Os sinais são capturados por uma placa de aquisição de dados desenvolvida no laboratório de eletrônica do IFBA. A operação do circuito bifásico era feita de forma a preestabelecer a fração de vazio desejada para a rodada experimental. Vazão de ar na corrente de agua do circuito. Foram testas 16 vazões de ar de acordo com a Tabela 1, sendo replicas nas vazões 7,9 $10 \mathrm{~mL} / \mathrm{min}$ de ar. A vazão de agua manteve-se constantes, colocava-se no sistema uma vazão de ar e a partir deste momento monitorava-se e fazia o processo de aquisição de dados.

Os modelos usados foram não lineares do tipo NARX polinomiais e neurais. Modelo foi composto com uma entrada, as vazões de ar introduzidas na corrente de água. A saída foram os sinais de voltagem medidas pelo sensor capacitivo. O sensor foi calibrado para água pura, quando a vazão de foi nula. 


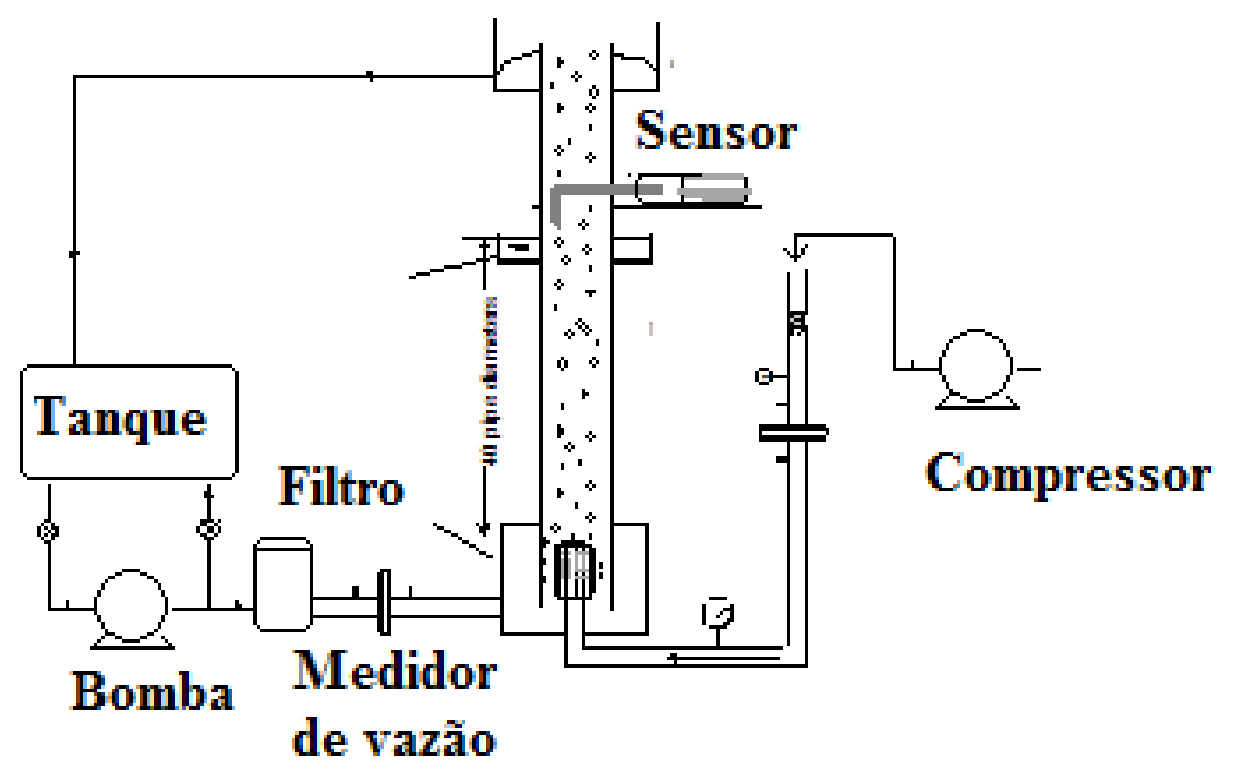

Figura 1 - Aparato experimental.

Tabela 1 - Dados utilizados na identificação do sensor para medição da vazão de ar.

Vazão de água (L/min)

1,5

1,5

1,5

1,5

1,5

1,5

1,5

1,5

1,5

1,5
Vazão de ar (mL/min)

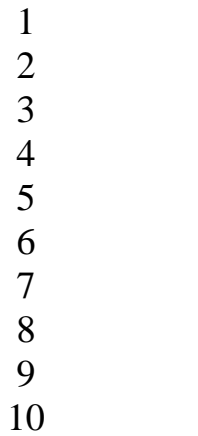

\section{RESULTADOS E DISCUSSÃO}

Os dados para os testes foram obtidos via aquisição com Labview e geraram vetores com 3000 linhas como é mostrado na Figura 3. Os dados foram obtidos fixando a vazão de água e variando a vazão de ar (conforme a Tabela 1). Os ensaios começaram com vazão nula de ar e colocando ar na tubulação até que a vazão atinge uma vazão máxima de ar, isso simulava uma perturbação degrau no sistema. Ao iniciar-se procedimento de identificação do sensor capacitivo, o seguinte procedimento foi estabelecido, o pré-processamento dos dados, entrada e a saída. A entrada dos dados é vazão de ar e sua saída sinal de voltagem lido pela placa de aquisição de dados. Para analise do sensor e seu 
projeto, os dados submetidos a filtros do tipo Fir e seus dados separados e foi montada uma nova planilha de modo que dados ficassem distribuídos de aleatoriamente. A Figura 2 apresenta esses dados depois de feito a separação e tratamento de filtragem, observa-se que houve uma diminuição dos níveis de ruídos. Os dados foram divididos em três blocos de 1000 linhas cada para que pudesse ser usados na rede neural.
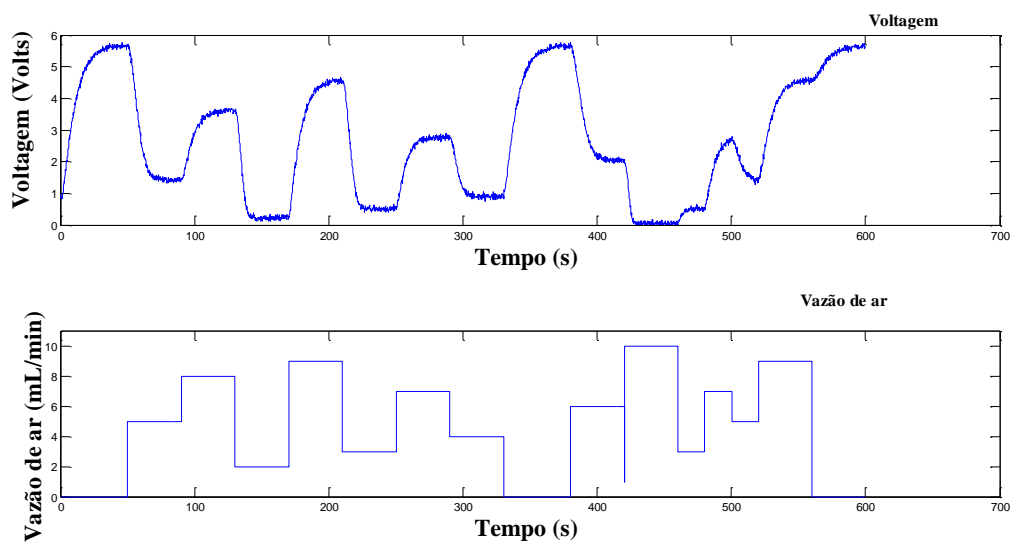

Figura 2 - Sinais de entrada a voltagem e os sinais de saída são a vazão de ar com tratamento.

A validação dinâmica do modelo não linear NARMAX é mostrada na Figura 3. A Figura 3 compara os dados medidos com dados estimados pelo modelo. Claramente observa-se que o modelo não linear reproduz a dinâmica do sistema. Os resultados mostram que há a possibilidade da presença de ruídos, repare que o modelo não consegue reproduzir alguns picos.

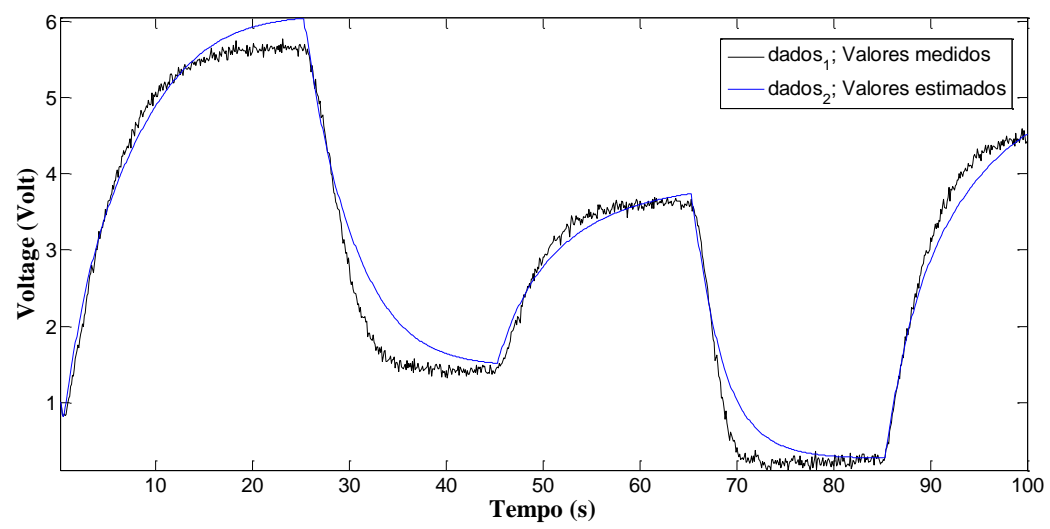

Figura 3 - Validação dinâmica com os dados utilizados para a detecção dos parâmetros.

Observa-se na Figura 4, que a curva dos valores reais e a dos valores estimados pelos neurônios da rede praticamente se sobrepõe, o que demostra precisão da estimação da rede neural. Depois de 
uma avaliação dos materiais e métodos usados, verificou-se então a viabilidade de sua aplicação no projeto de sensores virtuais reais. Tal resultado evidencia a utilização dessa rede no projeto de sensores virtuais reais. Observa-se que a rede neural elimina parte o ruído. Conforme o gráfico da Figura 6, o soft sensor apresentou bons resultados para estimar a voltagem da sonda capacitiva.

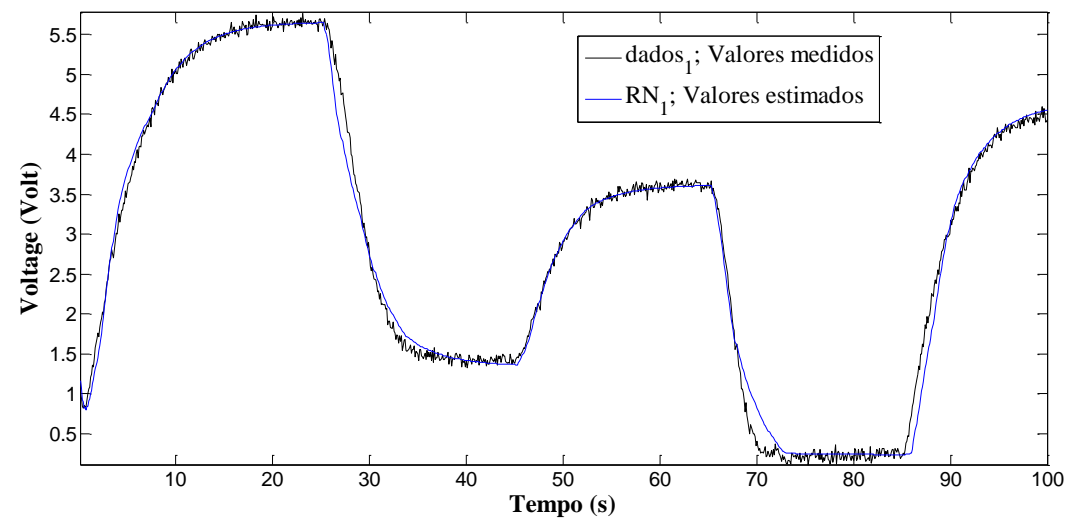

Figura 4 - Comparação do valor estimado com os valores medidos pela sonda capacitiva e rede neural para o soft sensor

A Figura 5 apresenta comparação entre a representação escolhida a NARMAX polinomial e a rede neural. Pela análise do gráfico da Figura 7, pode-se afirmar que os resultados obtidos com a NARMAX polinomial são inferiores aos resultados obtidos com a rede neural. Pois, os resultados obtidos com o NARMAX polinomial possuem uma maior variabilidade. Os resultados obtidos mostram que a abordagem proposta rede neural torna possível melhorar a capacidade de estimativa do modelo obtido com respeito a uma abordagem clássica NARMAX polinomiais (uma melhoria de cerca de $20 \%$ foi obtido com respeito ao modelo de referência). Uma comparação de dois métodos rede neural e NARMAX polinomial para abordagens do soft sensor revela que o melhor desempenho é obtida utilizando a rede neural. No entanto, a diferença entre estratégias de empilhamento não é tão relevante. 


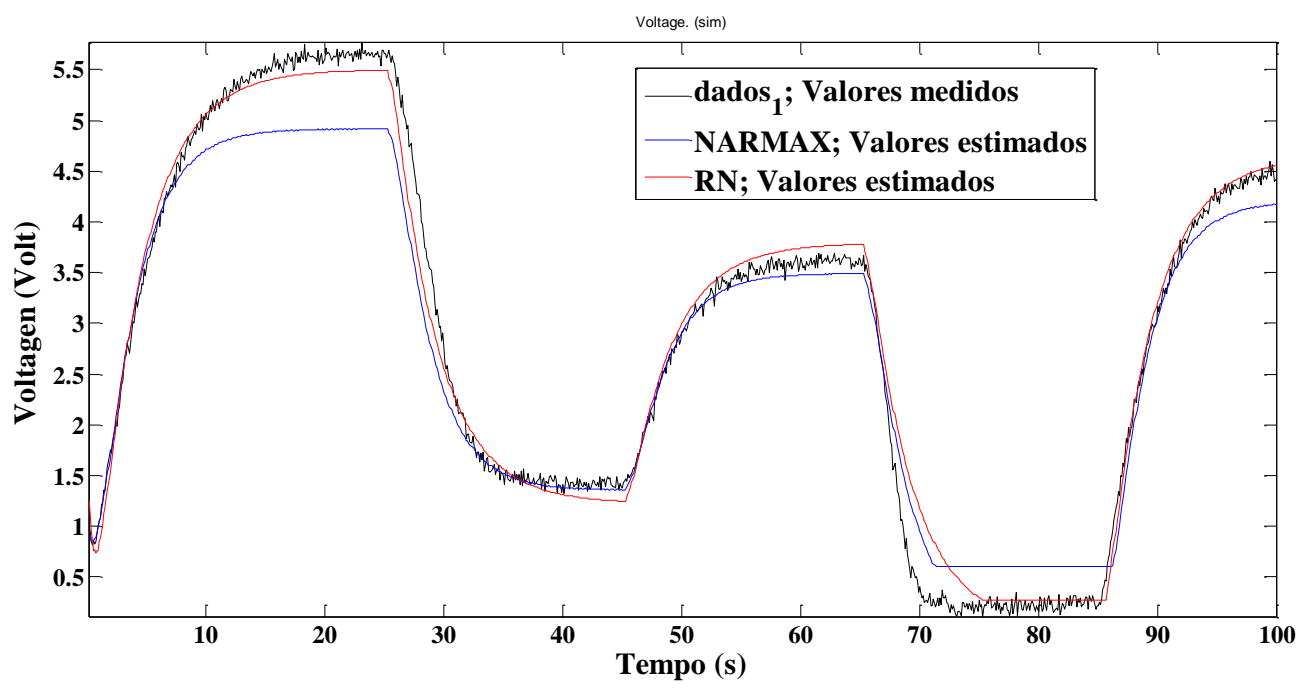

Figura 5 - Comparação do valor estimado com os valores medidos via NARMAX e rede neural pela sonda capacitiva para o soft sensor

\section{CONCLUSÕES}

O sensor virtual implementado por meio da rede neural e o método NARMAX polinomial mostraram-se de grande valia e confiabilidade, podendo ser utilizado tanto na medição da variável (numa inviabilidade de aquisição de um sensor físico) como também na intervenção durante uma falha ou manutenção desse sensor.

De acordo com os dados obtidos, tanto o método NARMAX polinomial e a rede neural apresentaram bom resultados. Sendo que, a rede neural apresentou um desempenho superior ao método NARMAX. No entanto, a diferença entre estratégias de empilhamento não é tão relevante.

\section{REFERENCIAS}

AGUIRRE, L.A. Introdução a Identificação de Sistemas - Técnicas Lineares e Não lineares Aplicadas a Sistemas Reais. $3^{\mathrm{a}}$ ed., UFMG, 2007.

ARAÚZO-BRAVO, M. J., CANO-IZQUIERDO, J. M., GÓMEZ-SÁNCHEZ, E., , “Automatization of a penicillin production process with soft sensors and an adaptive controller based on neuro fuzzy 
systems", Control Engineering Practice, v. 12, pp. 1073-1090, 2004.

ASSIS, A. J., MACIEL FILHO, R., "Soft sensorsdevelopment for on-line bioreactor state estimation", Computers and Chemical Engineering, v. 24, pp. 1099-1103. 2000.

FRANÇA, F. A., , As Transições Bolhas-Pistonado e Pistonado-Agitante em um Escoamento Bifásico Vertical Adiabático Ascendente, Tese de Doutorado, Unicamp, 1987

HEWITT, G. F. e ROBERTS, D. N., , Investigation of Interfacial Phenomena in Annular Two-PhaseFlow by Means of the Axial View Technique, AERE-R6070, 1982.

HUBBARD, M. G. e DUKLER, A. E., The Characterization of Flow Regimes for Horizontal TwoPhase Flow, Proc. Heat Transfer and Fluid Mechanics Inst., Stanford Univ. Press, California. 1966.

Linko, S., Zhu, Y-H. e Linko, P., Applying neural networks as software sensors for enzyme engineering, Trends in Biotechnology, vol.17, pp. 155-162, 1999.

MATSUI, G, Automatic Identification of Flow Regimes in Vertical Two-Phase Flow Using Differential Pressure Fluctuations, Nuclear Engineering and Design, 95, 221-231, 1986.

TAITEL, T.; BARNEA, D.; DUKLER, A. E. Modeling flow pattern transitions for steady upward gas liquid flow in vertical tubes. AIChEJ, [S.1.], v. 26, p. 345-54, 2, 70, 1980. 